\title{
Self-powering wireless devices for cloud manufacturing applications
}

\author{
V. Ostasevicius ${ }^{1} \cdot$ V. Jurenas ${ }^{1} \cdot$ V. Markevicius ${ }^{2} \cdot$ R. Gaidys ${ }^{1} \cdot$ M. Zilys ${ }^{2} \cdot$ M. Cepenas ${ }^{2}{ }^{\text {. }}$ \\ L. Kizauskiene ${ }^{3}$
}

Received: 9 April 2015 / Accepted: 19 July 2015 / Published online: 22 August 2015

(C) The Author(s) 2015. This article is published with open access at Springerlink.com

\begin{abstract}
Cloud manufacturing will play a significant role in future manufacturing, enabling companies to share resources and equipping them with scalable, flexible as well as costefficient manufacturing solutions with cheaper maintenance. With the main focus on cloud manufacturing, this paper aims to propose new devices for monitoring of manufacturing processes. The proposed self-powered wireless devices cover two main groups: rotating and non-rotating cutting tools. The most common rotating tool - mill — and non-rotating one - turning tool-are examined. Energy harvesting from the accelerations of the rotating cutting tool is mostly influenced by the speed of the tool and the number of cutting edges, while energy harvesting from the non-rotating tool is affected by the vibration modes of the tool. A significant difference between these two types of excitation frequencies obliges to use cantilever for energy harvesting from the rotating tool and disc-shaped piezoelectric harvesters for the non-rotating tool. The results of theoretical and experimental studies of the dynamics of these harvesters show an effective way for approaching their natural frequencies to the resonant frequencies of the cutting tools. The amplitude-frequency analysis of vibrations of the tool could be useful for the technological process monitoring as well as for the evaluation of machine tool state. Vibration and acoustic signal analysis using fast Fourier transform (FFT)
\end{abstract}

V. Ostasevicius

vytautas.ostasevicius@ktu.lt

1 Institute of Mechatronics, Kaunas University of Technology, Studentu St. 56-123, LT-51368 Kaunas, Lithuania

2 Faculty of Electrical and Electronics Engineering, Kaunas University of Technology, Studentu St. 48-211, LT-51368 Kaunas, Lithuania

3 Faculty of Informatics, Kaunas University of Technology, Studentu St. 48-213, LT-51368 Kaunas, Lithuania enables to identify the level of tool wear and, moreover, the mode of tool fixture and technical state of the spindle. As the intensity of energy accumulation depends on the state of the cutting tool wear, it indicates and detects the tool condition. The voltage generated from the cutting tool vibrations of the harvester exponentially rises till the capacitor is fully charged and a wireless signal is sent to the receiver. All the proposed technique and methods are inseparable from cloud manufacturing technologies.

Keywords Turning tool $\cdot$ Milling tool $\cdot$ Vibrations $\cdot$ Energy harvesting · Wireless transmission

\section{Introduction}

The recent prevalence of machine-to-machine serviceoriented computing and the emergence of cloud computing platforms as well as services provide promising new capabilities for wireless sensor networks (WSNs) [1]. Despite the issues in aggregating sensor data for the purposes of sharing in the form of 'big data' and for sensor fusion algorithm development [2,3], there are many potential advantages that can result from deploying WSNs. Individual sensor nodes can be installed on different parts of a machine tool and can be placed in remote locations. Moreover, it is also possible to retrofit sensors onto the already-installed machinery just slightly changing the machine configuration. Thus, the paper examines the potential use of such nodes and determines issues, closely related to achieving their effective implementation. The case studies correlate machine tool vibration with tool wear and were undertaken to show potential practical applications.

The aim and main focus of the paper lies on the extension of the useful lifetime of critical manufacturing elements, 
which can be achieved by estimating the lifetime of equipment, devices and their components. Improved sensors and sensor fusion have enabled a vast application of such technology for maintenance operations. As far as control of manufacturing is concerned, the current state of equipment can be determined from information on machine health, which also impacts the decisions on the control of the manufacturing system. This also enables supervisory controllers to take necessary actions in order to maintain undisrupted operation by employing fall-back strategies.

This paper seeks to determine the potential of wireless sensor nodes in manufacturing, describing specific case studies that provide the conditions to establish an infrastructure for monitoring based on MEMS accelerometer and applied for cutting tool vibrations. However, the analysis of vibrations is not the primary focus of the case study. Instead, the goal of the experiments is to demonstrate a new possible use of wireless sensors for the research of factory machinery and, more specifically, open-architecture machining systems, and their condition-based monitoring and predictive maintenance. Completed case studies demonstrate a linear relationship between tool wear and its vibrations.

Automation of manual processes, such as the collection of data on machine state or frequent sampling, is beneficial for predictive maintenance, just as more sensing points are useful for condition-based monitoring. Two specific areas of applying wireless sensors can be distinguished - motor analysis and performance of machine tools. The former is used to monitor the condition of small electric motors [4] and replace wires in traditional monitoring sensors of motor vibration [5], while the latter encompasses sensors for temperature measurement of end mill inserts [6] as well as monitoring based on vibrations to detect tool breakage [7]. To be more specific, machine tool lifetime is reduced by vibrations, which are caused by a number of factors, e.g. spindle characteristics, flexibility of structural elements, dynamic loading or conditions for cutting. The characteristics of such vibrations often include stability lobes which define the areas of safe machining [8].

Different from wired sensors, new application methods are possible with WSN, for example fusion of multi-sensor data by monitoring vibrations of a workpiece and/or spindle to determine tool wear, as well as wireless sensing of voltage, current and emission of acoustic signals [9]. WSN also facilitates new applications that earlier were impossible to achieve by wired sensors, such as more accurate multi-sensor condition monitoring techniques [10] and a hybrid network of remote wireless machine condition monitoring sensors and radio frequency identification tags that provide the technician a safe access on a mobile computer [11]. To a lesser extent, WSNs are also augmenting control systems in process manufacturing [12] and discrete manufacturing [13]. The general architectures consist of wireless sensors providing information to a wired control system that utilises sensor information and dispatches control signals to wired actuators, although prototype wireless actuators for low-latency process control also exist [14].

Nevertheless, applying wireless sensors to industrial applications presents several challenges as well. Predictive maintenance applications often require high-resolution data sampling, especially vibration analysis, but the computationally constrained WSN hardware platforms do not readily support such data-intensive sampling or provide sufficiently reliable end-to-end communication [15].

Cloud manufacturing is most often associated with the existing concept of networked manufacturing, which is also referred to as distributed manufacturing or Internet-based manufacturing [16]. However, the meaning of this concept is used in a much narrower sense nowadays, signifying the process of integrating distributed resources in order to complete one manufacturing task [17].

In contrast to the production-centred manufacturing, the service-centred type and the one enhanced by cloud computing can naturally gain much more benefit from cloud manufacturing. This is because cloud computing has evolved and converged a number of individual computing methods and approaches: Web 2.0, Internet delivery, virtualization, flexibility, distributed computing, grid computing, 'pay-asyou-go' service computing, content outsourcing, etc., to name a few [18]. To identify or, in other words tag, manufacturing resources, many technologies can be applied, for instance GPS, WSNs, sensor data classification, adapter technologies, clustering and analysis, Internet of things, radio frequency identification (RFID), computational RFID, cyber physical systems, etc. [19-21].

\section{Methodology}

Drilling, turning and milling are three main machining operations, while all the other processes are ascribed to various categories. In milling and drilling processes, the cutting edges are brought to bear against the workpiece due to the rotating cutting tool, and the turning process is the one that causes the workpiece to rotate. The first necessary condition for cloud manufacturing is related to the development of self-powered devices for these two groups of cutting tools. According to research [22], where the cutting tool is taken as an elastic structure having a number of natural vibration modes, the turning tool laterally vibrates on the main mode depending on the fixing, i.e. the length of the tool hanging from the claim, in a range from 2.5 to $5.6 \mathrm{kHz}$. To evaluate the possibility to harvest energy from the rotating tool, it was necessary to develop a special tool alimented from a battery device with an accelerometer and wireless transmission (Fig. 1a) as well as a receiver (Fig. 1b). 




a)



b)

Fig. 1 General view of the wireless rotating tool acceleration measurement device (a) and the receiver (b)

The angular vibrations of the milling tool holder were measured directly on the rotating tool. A pronounced periodicity was present in the acceleration measurements carried out during the milling operations. This periodicity can be directly associated with the harmonics of the tool structure, the number of cutting edges as well as the spindle speed $n$. Increasing the cutting depth resulted in increasing cutting forces and, thus, also in greater excitation levels. When changing the feed speed (feed rate), no significant changes were observed, although a small increase in the vibration level with increasing speed was noticed. The main purpose of using acceleration sensors was to discover any angular motion relating to torsional modes of the milling tool.

Milling can be described as an interrupted cutting process because during each revolution of the cutter, its teeth enter and exit the workpiece, and in this way, they are subjected to an impact force cycle. It can be seen from Fig. 2 that the $150-\mathrm{Hz}$ frequency dominates as a result of mill tooth and workpiece material collision. During this impact, the vibrations of the cutting tool are excited. The amplitudes of the vibrations between the interrupted cutting periods are considerably lower



Fig. 2 End milling tool vibrations under 3200-rpm four tooth mill

than during the impact and are characterized by lowered amplitudes till the new tooth comes in contact with the workpiece. These amplitudes are not sufficient for energy generation, and the most favourable are vibro-impact accelerations whose sequence intervals depend on cutting regimes. This means that higher effectiveness is demonstrated by the rotating cutting tool accelerations, mostly related to the tool speed and the number of cutting edges, rather than the cutting tool vibration modes. It is also claimed that energy harvesters for rotating and non-rotating cutting tools should be designed by using a few different types of piezoelectric transducers whose natural frequencies differ more than 10 times. In such a case, due to the force resulting from any changes in acceleration or motion, the mass squeezes the piezoelectric material, and it, in turn, generates an electrical charge corresponding to the force it was affected by. The initial conditions for energy harvester design are related to the necessity to find an appropriately shaped piezoelectric transducer for high and for sufficiently low frequencies. The first will be used for energy extraction from a non-rotating tool, while the second from a rotating one.

\section{Development of the energy harvesters}

One of the objectives of the paper is to find a shape of the piezoelectric harvester characterised by the given natural frequency and generating maximum power [23]. As the first mode shape of a fixed-free beam is close to the deflection curve subjected to a uniform load, such a piezoelectric cantilever load was chosen. In the case of linear deformations, the energy generated by piezo layers is proportional to the amount of deformation in this layer.

The equation of the deflection curve for a cantilever beam subjected to a uniform load of intensity $q$ is as follows:

$v=-\frac{q x^{2}}{24 E I}\left(6 L^{2}-4 L x+x^{2}\right)$,

where $E$ is the Young's modulus, $I$ the moment of inertia bending about the neutral axis, $L$ the length of the cantilever and $v$ the deflection. 
The deformations caused by the strains $\varepsilon_{x}$ are actual; thus, strain component $\varepsilon_{x}$ is analysed.

$\varepsilon_{x}=\frac{d^{2} v(x)}{d x^{2}}=-z\left(\frac{q x^{2}}{24 E I}\right)\left(12 L^{2}-24 x L+12 x^{2}\right)$.

The plane strain energy is determined by integrating over the upper plane of the beam:

$W=\frac{q^{2}}{(24 E I)^{2}} \frac{E W_{\mathrm{b}}}{2} \frac{H^{2}}{4} \int_{0}^{L} \varepsilon^{2} d x$,

where $W_{\mathrm{b}}$ is the width and $H$ is the height of the beam and uniform load $q$.

$W=\frac{q^{2}}{(24 E I)^{2}} \frac{E W_{\mathrm{b}}}{2} \frac{H^{2}}{4} \frac{144 L^{5}}{5}$.

The first natural frequency of the cantilever can be expressed as follows [24]:

$\omega=\frac{\lambda^{2}}{2 \pi} \sqrt{\frac{E}{12 \rho}} \frac{H}{L^{2}}, \lambda=1.8751$.

To choose the geometrical parameters of the cantilever, the optimization task can be expressed as

$$
\begin{gathered}
\operatorname{Max} W\left(L, W_{\mathrm{b}}, H\right) \\
\omega(H, L)=\omega^{0}, \\
L_{\min }<L<L_{\max }, \\
W_{\mathrm{b} \text { min }}<W_{\mathrm{b}}<W_{\mathrm{bmax}}
\end{gathered}
$$

From the frequency equation, $H$ expression is inserted into the equation for the calculation of energy, and thereby, the harvested energy will be calculated for the cantilever of the given natural frequency. The analysis was performed on a rectangular cross-section cantilever with the following dimensions: $L=0.0485 \mathrm{~m}, W_{\mathrm{b}}=0.0078 \mathrm{~m}, H=0.0007 \mathrm{~m}$, $E=7.5 \mathrm{e} 10 \mathrm{~N} / \mathrm{m}^{2}, \rho=7750 \mathrm{~kg} / \mathrm{m}^{3}$ and natural frequency $\omega^{0}=149.5 \mathrm{~Hz}$. The coefficient of stiffness of the cantilever can be calculated as $K=3 E I / L^{3}$ and the equivalent distributed charge is $q=q_{0} 8 E I / L^{4}$, where $q_{0}=1$. The variation of the cantilever dimension diapasons is $1 \mathrm{e}-2 \mathrm{~m}<L<4 \mathrm{e}-2 \mathrm{~m}, 2 \mathrm{e}-3 \mathrm{~m}<$ $W_{\mathrm{b}}<1.2 \mathrm{e}-3 \mathrm{~m}$. As can be seen from Fig. 3, the biggest strain energy is generated by the cantilever whose width and length proportion gains the upper limits of constraints.

Figure 3 demonstrates that the amount of energy from the cantilever length is non-linear as compared to width-linear; thus, the non-linearity of wider cantilevers is bigger.

Figure 4 presents the dependence of energy field values on the length and width of the cantilever, which allows properly selecting the dimensions of the cantilever assuring the maximum amount of the delivered energy. It is clear that the



Fig. 3 The dependence of resilient energy on the length and width of the cantilever when natural frequency is fixed and normalised for $(0.1)$ interval

thicknesses of the cantilevers are different, but the frequencies are equal.

As the search for the shape of the piezoelectric transducer of the rotating tool indicates the structure of the cantilever, the Noliac piezoelectric transducer CMBP04 was chosen. It can be described as a co-fired multi-layered piezoelectric transducer requiring low voltage, capable of converting a bending movement into an electrical output, and normally applied when a large displacement can be caused by a low force. Figure 5 shows the full architecture of the wireless energy generator.



Fig. 4 The dependence of the cantilever resilient energy on the cantilever length and width when natural frequency is given when the length and energy are normalised for (0.1) interval widths according to length 




Fig. 5 The architecture of the wireless vibration energy generator: 1 type of the cantilever: Noliac CMBP piezoelectric transducer, 2 concentrated inertial mass for the tuning of resonant frequency, 3 connection unit, 4 controller, 5 electrical energy accumulator, and 6 wireless transmitter

To increase the effectiveness of energy harvesting and sensitivity of the piezoelectric transducer 1 , the resonant frequency of transverse vibrations $F_{\mathrm{k}}(t)$ is tuned by changing the concentrated inertial mass 2 and approaching it to the frequency of $150 \mathrm{~Hz}$ excited by cutting forces $F_{\mathrm{p}}(t)$. As the Noliac CMBP piezoelectric transducer is composed of two layers, the resonant frequency of the cantilever-type piezoelectric transducer can be changed by connecting the appropriate shunt resistance to one of these layers (Fig. 6).

As Fig. 6 suggests, it is possible to change the resistance slightly and the amplitudes of the generated voltage considerably. The general view of the implemented prototype of the wireless energy harvester together with the diagnostic signal receiver is presented in Fig. 7.

For the turning energy harvesting, a disc-shaped piezo element was used. Under uniform pressure $q_{0}$, the disc deflection $W$ can be expressed as follows:

$W=\frac{q_{0}}{64 D}\left(R^{2}-r^{2}\right)^{2}$,



Fig. 6 Amplitude-frequency characteristics of the electric signal of the cantilever without concentrated inertial mass piezoelectric transducer when shunt resistance is infinite (open circuit-continuous line) and when one layer is shunted (short circuit—dotted line)

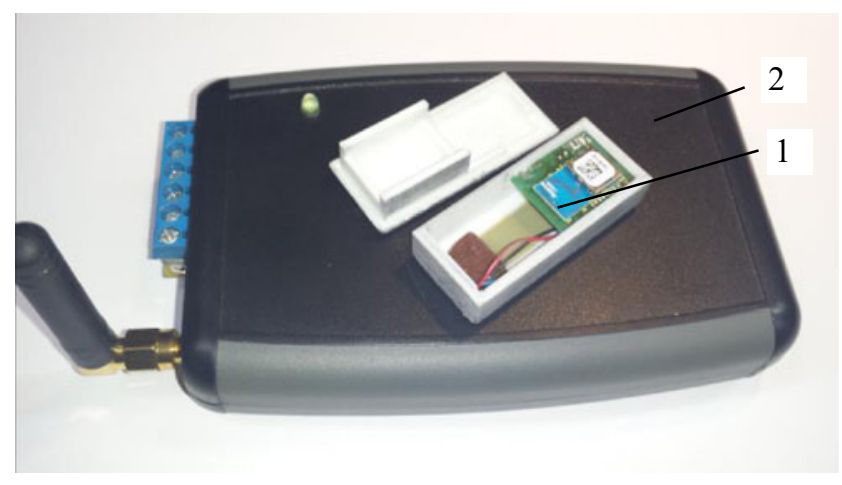

Fig. 7 General view of the electrical energy harvester with the energy accumulation, wireless transmitter ( 1 ) and diagnostic signal receiver (2)

where:

$\begin{array}{ll}R & \text { disc radius } \\ D=E h^{3} / 12\left(1-\mu^{2}\right) & \text { flexural rigidity } \\ E & \text { Young's modulus } \\ h & \text { thickness of the plate } \\ \mu & \text { Poisson's ratio. }\end{array}$

Next, the relation between strain and displacement of the circular plate with axisymmetric vibrations is calculated in such a manner:

$\varepsilon_{\mathrm{r}}=-z d^{2} w / d r^{2}$

where $\varepsilon_{\mathrm{r}}$ is the radial strain and $w(r)$ the out-of-plane transverse deflection of the middle surface.

$\varepsilon_{\mathrm{r}}=\frac{q_{0}}{64 D}\left(-4 z\left(3 r^{2}-R^{2}\right)\right)$.

Equations (10) and (11) give the expression of the strain energy $U$ of the plate caused by bending the axisymmetric circular plate with an initial deflection:

$U=\frac{q_{0}}{64 D} \int_{0}^{R}\left(\frac{\partial^{2} w}{\partial r^{2}}\right)^{2} r d r$

or

$U=\pi \frac{q_{0}}{64 D} 8 R^{6}$.

The first natural frequency of the circular plate with clamped edge is as follows:

$\omega=\lambda^{2} \sqrt{\frac{D}{\rho h}}$,

where $\lambda^{2}=10.215$ and $\rho$ is the mass density.

Figure 8 shows that the dependence of the amount of the generated energy in the disc plane on the width of the disc is linear, while the dependence of the generated energy on the 
Fig. 8 The dependence of the amount of generated energy on the geometric parameters of the disc-shaped piezo harvester: radius (continuous line) and width (dashed line), under the fixed natural frequency



disc radius is non-linear. In both cases, the biggest amount of energy is generated by the disc with larger geometrical dimensions.

Commercial Noliac CMBP piezoelectric transducers are not manufactured for a non-rotating tool because of very low resonant frequency. Therefore, circular piezo transducer bimorphs that are made of a piezo element and a bronze disc and have the necessary resonance frequency diapason were used. A mathematical model was created to analyse the possibility to apply frequency tuning to the piezoelectric transducer by using COMSOL multiphysics as a FEA tool, since this is one of the objectives of this paper. Hence, as Fig. 9a shows, the model of the piezoelectric transducer bimorphs (a) is composed of a brass disc (diameter $15 \mathrm{~mm}$, width $0.1 \mathrm{~mm}$ ) and a piezoelectric disc (diameter $10 \mathrm{~mm}$, width $0.1 \mathrm{~mm}$ ).

During the simulation, the fixture was made like a spring foundation having damping losses and the electrical borders like $10 \mathrm{~m} \Omega$ load of resistor. Then, the model was linked with loose tetrahedral elements of brass base and loose triangular swept mesh of piezoelectric part.

It is really complicated to use the capability of tuning the natural frequency, but in order to attain this effect, the suggested method was the relocation of the circumferential fixture with the one having a smaller diameter. Thus, the fixture was made into a ring-shaped spring foundation of $0.5 \mathrm{~mm}$ width on a foundation of the transducer. Mode frequency analysis (Fig. 9b) reveals that the resonant frequency initially increases because of the fixture radius reduction, but then the frequency begins to drop approximately at $0.7 r / R$ of size ratio. If the excitation frequency is defined earlier, it is possible to choose thoroughly the geometry and dimensions of a conventional linear harvester that fit to its excitation frequency and resonant frequency. The application of the circular piezoelectric bimorph (Fig. 10), including the option of approaching the frequency of piezoelectric bimorph to the frequency of the cutting tool, helped to develop a self-powered wireless node of the sensor for the cutting tool. The developed advanced wireless sensor architecture with separate ultralow power voltage detector and elaborated algorithm could reach only 100 $150 \mathrm{nA}$ triggering current after the capacitor is charged. Stateof-the-art nano-watt triggering power consumption allows to accumulate energy from very low energy sources, wake up an embedded microsystem and transfer sensor status without an additional energy source.

\section{Transducer model}

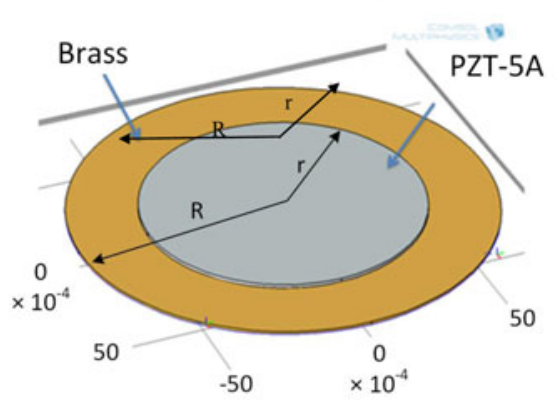

a)

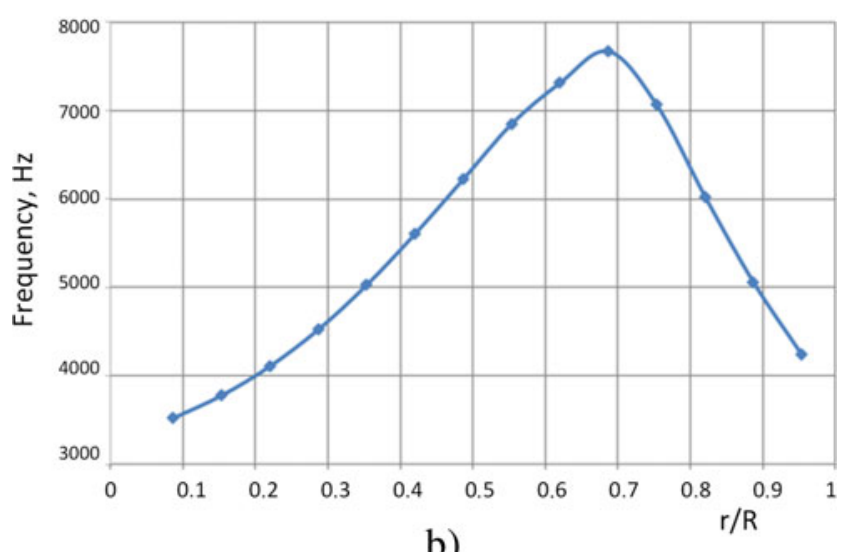

Fig. 9 Diagram of the circular piezo transducer bimorph (a) and a chart of dependence of natural frequency on fixture size ratio $r / R(\mathbf{b})$ 


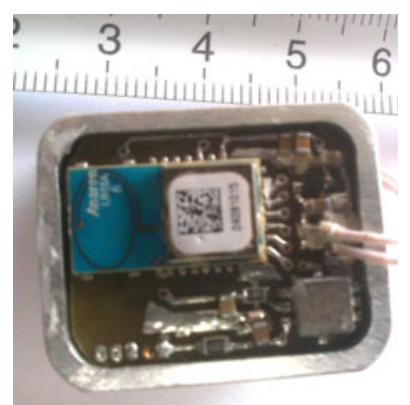

Fig. 10 Self-powered wireless node of the sensor used with a turning tool

These energy-harvesting prototypes for rotating (Fig. 7) and non-rotating (Fig. 10) tools fully satisfy the energy needs for sensor supply and are capable to transmit information at the distance of $20 \mathrm{~m}$. Two types of receivers for wirelessly transmitted information from sensors were elaborated (Fig. 11).

\section{Experimental procedure}

Installation of a monitoring system into an industrial manufacturing process can be necessary for various reasons. Due to redundant wear of cutting tools, distortions of

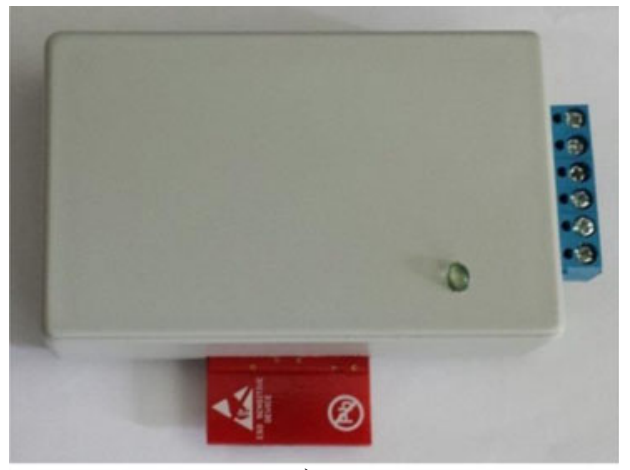

a)



b)

Fig. 11 Prototypes of wireless sensor node receiver 1 with a surface mount antenna and $\mathbf{b}$ separate antenna 2



Fig. 12 Turning on $\mathrm{CNC}$ lathe RAYO 165 process monitoring: 1 workpiece, 2 turning tool, 3 wireless sensor node with the energy harvester, 4 receiver, and $5 \mathrm{PC}$ with information proceeding program

dimensions of the produced components arise and this often increases scrapped levels, sustaining extra expenses. Signal components of the parameters of the cutting process which can be treated as suitable to detect and monitor tool wear can be recognised using the data which was derived from a number of experimental test cuts. The analyses of the signal clearly show correlation between the chosen signals and the cutting tool wear. It is possible to detect the changes of sensor signals in the vibration signatures in the course of the machining operation process.

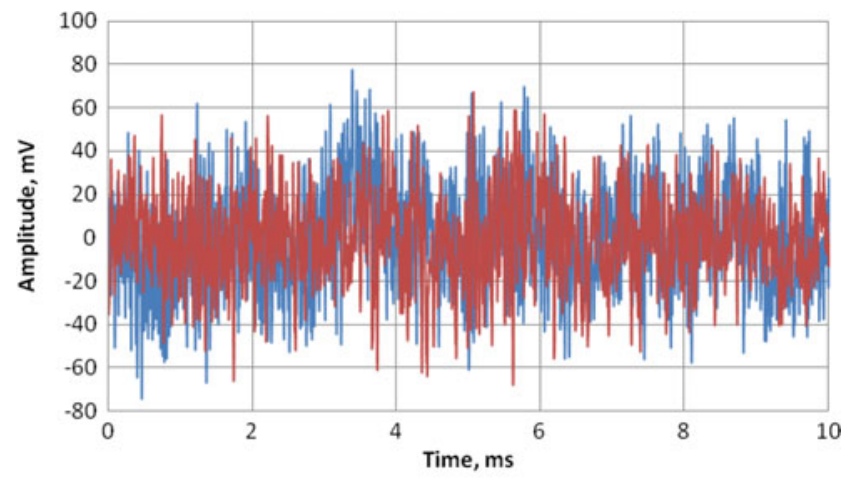

a)



b)

Fig. 13 Turning tool vibration cutting steel with the new (a) and the worn (b) tools (blue colour - axial vibrations, red colour - transversal vibrations) 


\subsection{Turning process vibrations}

The concept of cutting vibration energy harvester was demonstrated for steel turning operation on CNC lathe RAYO 165, where the depth of the cut was $0.5 \mathrm{~mm}$. Figure 12 demonstrates the experimental energy-harvesting setup.

The created wireless sensor node was mounted on the CNC turning tool 2, and the harvested energy was accumulated by a $100-\mu \mathrm{F}$ capacitor. The aim of the experiment was to reveal the influence of the tool wear on the tool vibration amplitudes. A correlation was detected between the vibration acceleration amplitude and the tool wear. The analysis of vibration signature in the course of the cutting process is proven as a useful method for the detection of the tool wear. Thus, the analysis of the measurements revealed (Fig. 13) the possibility to use the analysis of vibration acceleration amplitudes directly for the assessment of the tool wear. When the cutting time increases,



a)



b)

Fig. 14 Turning tool vibration FFT (a) and vibration frequency distribution (b) cutting steel with the new tool



Fig. 15 Experimental setup for the diagnostics of acoustical signal during turning: 1 microphone of the acoustic measurement device, 2 zone of turning process, 3 controller of acoustic signal, and 4 PC with the acoustic signal analysis program

the signal of acceleration differs, but this was not visible on the plots of time domain because bigger frequency components



a)



b)

Fig. 16 Typical AE signals in turning: a new tool and $\mathbf{b}$ worn tool 
hid the smaller ones, but the full spectra could be observed in the frequency domain. The time domain also clearly demonstrated that that the signals reacted in a clearly accidental way and showed dynamic properties. The results of the measurements show that vibration amplitudes of the worn tool increased 3 times in axial and 5 times in transversal directions.

To assess the vibration signatures and the signals of dynamic cutting force, the measured signals had to be transformed from time to frequency domain using fast Fourier transform
(FFT). Figure 14 shows the turning tool vibration FFT and frequency distribution cutting steel with the new tool.

This method enabled to reveal the properties or specific information of the cutting process covered up into the time domain and to start the analysed and produced spectra with properties characterizing the tool at the initial contact (i.e. with a new and sharp tool). Then, it was finished by defining the spectra of properties of an apparently worn tool (or the random failure/chipping modes). The results of the spectra enabled to
Fig. 17 FFT applied to AE signal of the turning tool cutting steel with the new (a) and worn (b) tool

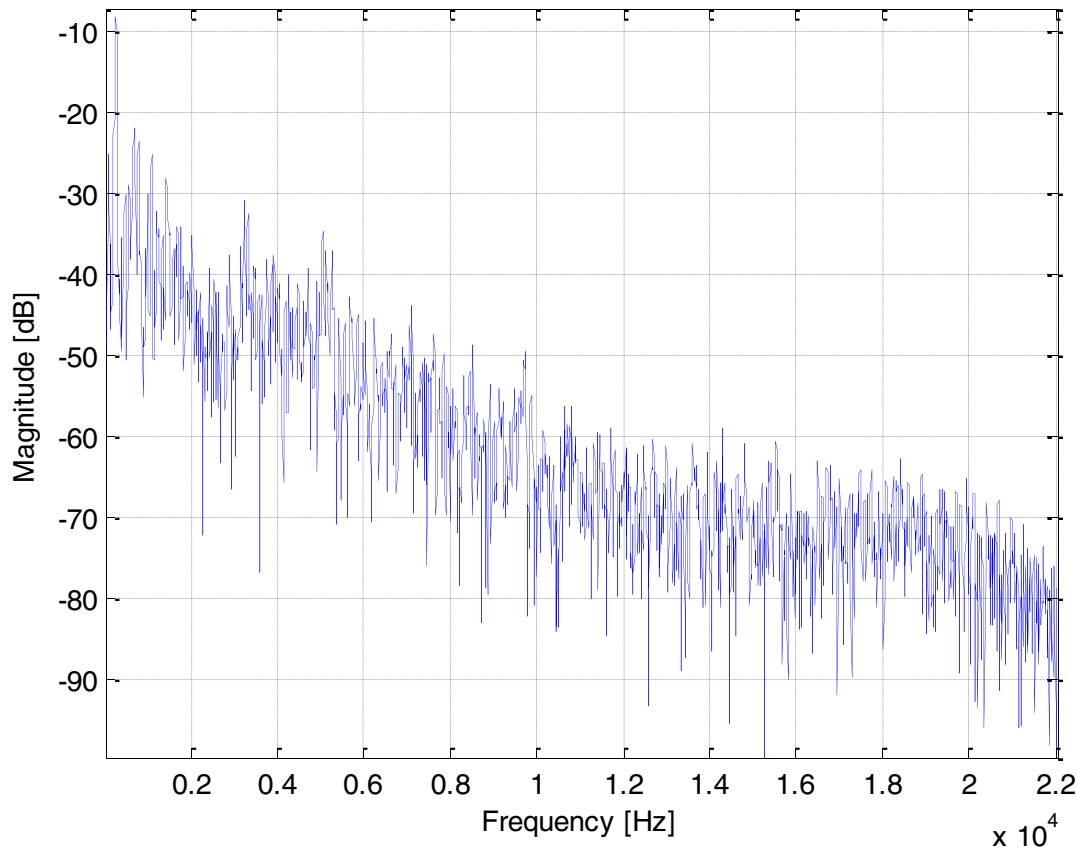

a)



b) 
detect a distinctive difference of signal characteristics once the cutting process was carried out using a new insert in comparison to the other one done with a damaging degree of wear. The specific amplitude growth of the principal resonance frequency peak of the intense force signal was referred to the periodic stick-slip effect within the contact area. The physics of this effect can be explained by the sharp edges of a new tool that reduced the contact between the tool and the workpiece. The growing degree of wear caused a raise of the contact area as a result of deteriorated cutting edges. High signal pulses that are developed by the friction transition from steady to floating influence the growth of the principal resonant peak magnitude and the emergence as secondary peaks at the band region of about $10 \mathrm{kHz}$. Having reached a particular wear value of the flank, the principal frequency peak becomes damped when the contact area evenly expands, partially because of the growth of contact friction, its plastic deformation and the growth of the further nose wear. Thus, it is possible to demonstrate how the development of the even nose wear leads to a drop in the resonant peak magnitude. The control of the shaped chips showed that the high burst was caused by a growth of the lamination frequency (from 0.1 to $10 \mathrm{kHz}$ approximately). The growth of the lamination frequency was caused by the alteration of the material deformation behaviour on the shear zone. It is possible that the decrease in the peak amplitude of resonant frequency due to a strong wear of the tool is caused by the growth of the rake angle from the friction effect of the rough chip.

\subsection{Acoustic emission of the turning process}

The device for measuring acoustic emission (indicated by 1 in Fig. 15) was elaborated for evaluating the reliability of vibrational analysis.

Acoustic emission (AE) belongs to the group of phenomena that develops transient elastic waves using the rapid release of energy from a fixed source or sources in a material, or the transient elastic wave(s) generated in this way. Using $\mathrm{AE}$ for controlling tool condition is the reason that $\mathrm{AE}$ signal frequency range is considerably greater than that of the environmental noises and machine vibrations, and does not interrupt the operation of cutting. According to the results of the research, AE of the stress waves, derived from the abrupt release of energy in deforming materials, was effectively applied in laboratory tests to trace tool wear during the operations of the single point turning.

On the basis of the analysis of AE signal sources, AE generated from metal turning comprises the permanent and transient signals having completely diverse characteristics. Permanent signals are related to shearing within the fundamental zone and wear on the flank and tool face, whereas transient signals or bursts are derived from chip fracture or tool breakage (Fig. 16).
An AE signal is non-fixed and frequently consists of overlapping transients with unknown waveforms and times of arrival. The biggest problem of processing an $\mathrm{AE}$ signal is to obtain proper physical parameters, like tool wear, when these parameters include variations of frequency as well as time.

FFT was used to analyse signals of AE (Fig. 17) and identify frequency diapasons (Fig. 18).

The graphs in Fig. 18 show that the turning process using the new tool with an acoustic signal is characterised by low vibration amplitudes (0.05) and an even frequency spectrum. In contrast, the worn tool raises the noise level by $5-6$ times and develops higher resonance modes at 10 and $15 \mathrm{kHz}$ in the frequency spectrum beyond the first mode at $5 \mathrm{kHz}$.

Distribution of acoustical signal in the turning operation of steel with the new (Fig. 18a) tool could be characterised by the



a)



b)

Fig. 18 Distribution of acoustical signal in turning operation of steel with the new (a) and worn (b) tool 
low amplitude (approximately $0.05 \mathrm{~mm}$ ) and quite smooth frequency spectrum, as well as in turning with the worn tool (Fig. 18b), the vibration amplitude becomes by $5-6$ times higher and the first (approximately at $5 \mathrm{kHz}$ ) and higher (at 10 and $15 \mathrm{kHz}$ ) modes could be recognised from the frequency spectrum.

\section{Milling process}

The developed wireless piezoelectric energy harvester with a subsystem of wireless information transfer and a subsystem of energy accumulation was applied for steel milling operation on Deckel Maho DMU 50 Ecoline CNC universal machining centre shown in Fig. 19.

The experiment was done on a steel workpiece with end mill working regimes: mill speed $-3200 \mathrm{rpm}$, feed$0.1 \mathrm{~mm} / \mathrm{rev}$, and depth of the cut $-1 \mathrm{~mm}$. Two kinds of mills were chosen: one was a new mill and the other was worn out. These two mills were expected to show different results: predictably, the worn mill would have higher resonance frequencies due to higher vibrations. A simple $1-\mathrm{mm}$ depth, 5-mm milling edge of the tool on the workpiece, with the feed rate of $600 \mathrm{~mm} / \mathrm{min}$ and $3200 \mathrm{rpm}$, was chosen, and a 'zigzag' milling principle was applied. Fast Fourier transform of mills vibration characteristic is presented in Fig. 20.

As Fig. 20 shows, the dominant frequencies of the end mill are $150-212 \mathrm{~Hz}$ and its multiples.

Further experiment was organised to show the influence of the tool wear on the frequency of wireless information



Fig. 20 Amplitude-frequency characteristic of end mill vibrations at $3200 \mathrm{rpm}$

transmission to the receiver (Fig. 21). During the milling operation, a piezo harvester was excited by the mill vibrations and the harvested energy was accumulated by a $100-\mu \mathrm{F}$ capacitor.

Figure 21 suggests that the maximum output voltage of the vibration harvester when cutting by the new tool is $2 \mathrm{~V}$ (blue colour). The generated voltage of the harvester was exponentially rising till the capacitor was fully charged, and a wireless signal is sent to the receiver. The signal sending time intervals amounted to $\sim 6 \mathrm{~s}$. The accelerometer signal is marked in red colour.

When the tool life comes to an end and the cutting edge of the tool is worn, the signal amplitude of the accelerometers (Fig. 22) as well as the frequency of wireless transmission of the measured results increases, while the time interval is decreased by approximately $2-3$ times till $\sim 2-3$ s.
Fig. 19 a General view of Deckel Maho DMU 50 Ecoline CNC universal machining centre, b four teeth end mill (diameter $40 \mathrm{~mm}$ ), and $\mathbf{c}$ general view of end mill vibration harvesting: 1 workpiece, 2 end mill, and 3 piezoelectric energy harvester with a subsystem of wireless information transfer and a subsystem of energy accumulation



a)



b)



c) 




Fig. 21 Exponentially rising generated voltage of the harvester (upper blue colour) and the signals of the new tool vibrations (lower red colour) when milling a steel workpiece; the time of capacitor charge till $2 \mathrm{~V}$ is $\sim 6 \mathrm{~s}$

\section{Applicability of the created devices for cloud manufacturing}

Manufacturers are harnessing ever-increasing amounts of data from equipment and suppliers to improve decisionmaking. The ability to analyse data from every link of the manufacturing value chain helps companies to better control manufacturing processes, keep production running smoothly and reduce costs. Monitoring is an important part of manufacturing process control and management. It plays a crucial role in ensuring agility in a manufacturing system, process robustness, responsiveness to client demands and achievement of a sustainable production environment. Recent developments in information systems and computer technology allow for the implementation of new philosophies that integrate various monitoring applications into one complex system connected through company-wide IT systems and with systems operating throughout the whole supply chain. Accessing manufacturing and other industrial data in the cloud via wireless devices such as self-powering sensors provides many benefits.



Fig. 22 Exponentially rising generated voltage of the harvester (upper blue colour) and the signals of the worn tool vibrations (lower red colour) when turning a steel workpiece; the time of capacitor charge till $2 \mathrm{~V}$ is $\sim 2-$ $3 \mathrm{~s}$
The most efficient way for monitoring the technological processes is related to the cloud manufacturing possibilities, where not only appropriate software but the attention of highlevel specialists could be found. The idea behind cloud manufacturing processes, including the involved components and the useful cloud-based services, is presented in Fig. 23. The upper layer, denoted in the picture, represents the factory floor setting, the deployed machinery, equipment and smart tools, as well as the final consumers of the cloud services, i.e. the factory employees or other industrial partners. Wireless sensor nodes mounted on various parts of machine tools transmit the sensed data to the communication devices, through which the collected data is further transmitted to cloud resources for further processing. The cloud infrastructure and services are provided by the third party companies, assuring the customer enterprises unlimited amount of the required storage and processing resources as well as the applications and most acceptable pricing and usage options. The beneficial cloud services could be mainly focused on the monitoring and control of the manufacturing process: the predictive maintenance and condition-based monitoring of the technological processes, the FFT for vibration and acoustic signal analysis or even real-time automatic control of the devices. The information about the cutting regimes and machine tool characteristics could be easily presented for the end users, assuring not only the preferred visual representation of the results but the variety of end-user devices as well. For the complete evaluation of technological process by every industrial partner, other special software and qualified specialists would be available too.

\section{Conclusions}

This paper demonstrates cloud-based production monitoring devices covering two main cutting tool groups: rotating and non-rotating. The different energy-harvesting possibilities from these two cutting tool types necessitated the investigation of appropriate piezo transducers for low-level $150-200-\mathrm{Hz}$ and high-level 4.0-4.5-kHz excitation frequencies. The developed low power wireless sensor nodes with energy-harvesting techniques have the ability to accurately monitor the condition of the tools. The harvester consisting of a piezoelectric transducer utilises vibrations of the cutting tool to collect the mechanical power out of the cutting process and then transform it into electrical power. Moreover, through the use of the rectification and energy storage circuit, it is possible to use the accumulated energy to permanently power the sensors as well as the wireless module. The integration of the sensors, wireless module and an accelerometer enabled the development of a selfpowered wireless system for vibration sensing, which was necessary for the evaluation of the manufacturing process conditions by sending information wirelessly to the Internet or Wi-Fi 




Fig. 23 Cloud-based services application for manufacturing

applications. The cutting tool wear influences the level of vibrations during the manufacturing process. To assess the performance of the cutting tool, it is necessary to define the critical moment when the cutting tool starts to manufacture parts of inappropriate quality, and the best way to carry out such an assessment is to measure vibrations or acoustics. The proposed technique is applicable for a cloud manufacturing environment. The result of the system monitoring during the cutting process revealed that the system can efficiently sense the vibrations generated by the cutting tool, then transmit its signals to the terminal computer and create the necessary conditions for jumping into a cloud computing initiative adopted in the manufacturing sector.

Acknowledgments This study was supported under the EU Eurostars project E! 7288 MOINFO (Mobile-Based Tool for Mechanical Manufacturing Equipment).

Open Access This article is distributed under the terms of the Creative Commons Attribution 4.0 International License (http://creativecommons.org/ licenses/by/4.0/), which permits unrestricted use, distribution, and reproduction in any medium, provided you give appropriate credit to the original author(s) and the source, provide a link to the Creative Commons license, and indicate if changes were made.

\section{References}

1. Dargie W, Poellabauer C (2010) Fundamentals of wireless sensor networks: theory and practice. Wiley, West Sussex, 330 p

2. Cuzzocrea A, Fortino G (2013) Managing data and processes in cloud-enabled large-scale sensor networks: state-of-the-art and future research directions. In: 13th IEEE/ACM international symposium on cluster, cloud, and grid computing: 583-588

3. Govindan R, Hellerstein JM, Hong W, Madden S, Franklin M, Shenker S (2002) The sensor network as a database. University of Southern California: 1-8

4. Lu B, Habetler TG, Harley RG, Gutierrez JA, Durocher DB (2007) Energy evaluation goes wireless. IEEE Ind Appl Mag 13(2):17-23

5. Jagannath VMD, Raman B (2007) WiBeaM: wireless bearing monitoring system. In: Communication systems software and middleware, COMSWARE, 2nd international conference: $1-8$

6. Wright P, Dornfeld D, Hillaire R, Ota N (2006) Tool temperature measurement and its integration within a manufacturing system. Trans of NAMRI/SME 34:63-70

7. Sudararajan V, Redfern A, Schneider M, Wright P (2005) Wireless sensor networks for machinery monitoring. In: ASME International Mechanical Engineering Congress and Exposition

8. Altintas Y, Budak E (1995) Analytical prediction of stability lobes in milling. Ann CIRP 44(1):357-362

9. Ghosh N, Ravi YB, Patra A, Mukhopadhyay S, Paul S, Mohanty AR, Chattopadhyay AB (2007) Estimation of tool wear during 
CNC milling using neural network-based sensor fusion. Mech Syst Signal Process 21(1):466-479

10. Jardine AKS, Lin D, Banjevic D (2006) A review on machinery diagnostics and prognostics implementing condition-based maintenance. Mech Syst Signal Process 20(7):1483-1510

11. Ramamurthy H, Prabhu BS, Gadh R, Madni AM (2007) Wireless industrial monitoring and control using a smart sensor platform. IEEE Sensors J 7(5-6):611-618

12. Koumpis K, Hanna L, Andersson M, Johansson M (2005) Wireless industrial control and monitoring beyond cable replacement. In: Profibus international conference. Coombe Abbey, Warwickshire, UK

13. Korber HJ, Wattar H, Scholl G (2007) Modular wireless real-time sensor/actuator network for factory automation applications. IEEE Trans Indust Inform 3(2):111-119

14. Johnstone I, Nicholson J, Shehzad B, Slipp J (2007) Experiences from a wireless sensor network deployment in a petroleum environment. In: Proceedings of international conference on wireless communications and mobile computing, Honolulu

15. Krishnamurthy L, Adler R, Buonadonna P, Chhabra J, Flanigan M, Kushalnagar N, Nachman L, Yarvis M (2005) Design and deployment of industrial sensor networks: experiences from a semiconductor plant and the North Sea. In: ACM SenSys
16. Xu X (2012) From cloud computing to cloud manufacturing. Robot Comput Integr Manufac 28(1):75-86

17. Pallis G (2010) Cloud computing: the new frontier of internet computing. IEEE Internet Comput 2010:70-73

18. Foster I, Zhao Y, Raicu I, Lu S (2008) Cloud computing and grid computing 360 degree compared. In: Grid computing environments workshop

19. Kunio T (2010) NEC cloud computing system. I: NEC Tech J 5(2): $10-15$

20. Dikaiakos MD, Katsaros D, Mehra P, Pallis G, Vakali A (2009) Cloud computing: distributed internet computing for IT and scientific research. IEEE Internet Comput 13(5):10-11

21. Ryan MD (2011) Viewpoint cloud computing privacy concerns on our doorstep. Commun ACM 54(1):36-38

22. Ostasevicius V, Gaidys R, Rimkeviciene J, Dauksevicius R (2010) An approach based on tool mode control for surface roughness reduction in high-frequency vibration cutting. J Sound Vibrat 329(23):4866-4879

23. Ostasevicius V, Milasauskaite I, Dauksevicius R, Baltrusaitis V, Grigaliunas V, Prosycevas I (2010) Experimental characterization of material structure of piezoelectric PVDF polymer. Mechanika 6:78-82

24. Rao SS (2007) Vibration of continuous systems. Wiley, New York, $720 \mathrm{p}$ 